\title{
Identification of Edible and Non-Edible Mushroom Through Convolution Neural Network
}

\author{
Devika $\mathrm{G}^{1, *}$ Asha Gowda Karegowda ${ }^{2}$ \\ ${ }^{I}$ Government Engineering College, KR Pet, Karnataka, India, sgdevika@gmail.com \\ ${ }^{2}$ Siddaganga Institute of Technology, Tumakuru, Karnataka, India, \\ *Corresponding author. Email: sgdevika@gmail.com
}

\begin{abstract}
Mushroom is one among the most popular consumed food in India. In India people are cultivating mushroom as viable income source for their livelihood. Now-a-days deep learning is being applied to process big data and vision related applications. Recent smart devices can be utilized for automated edibility diagnosis of mushroom using deep convolution neural network $(\mathrm{CNN})$ it has revealed a remarkable performance capability in all its sphere of research activities. DCNN works on static dataset. The models on which it applies will pose as well determine its requirement for training. This paper presents a classification tool for edibility detection of mushroom through deep CNN. Better performance is obtained by tuning the hyper-parameters and through adjustments in pooling combinations in order to obtain real time inference suitably. DCNN has been trained with a data set of segmentation as train and test sets. Performance is analyzed on sNet, Lenet, AlxNet, cNET network architectures. DCNN results are comparatively better in its performance.
\end{abstract}

Keywords: Bigdata, CNN, classification, DCNN, Mushroom,

\section{INTRODUCTION}

Mushrooms have origin from fungi family. A viewable sized fruit body produced by fungi is known as mushroom. They are not autotrophs with the absence of chlorophyll but through their enzymes can degrade complex substrate to get nutrition required for growth [1-3]. They are capable to flourish in all wide range of habitats from tropics to polar and from below to above soil. There is no typical method for classification of mushrooms. In general, they are categorized as edible or medicinal or poisonous mushrooms. Based on habitat mushrooms are categorized as temperate, tropical or sub-tropical mushrooms. On morphological features grouped as grilled or others (Cyphelloids, Gasteroids, Clavarioid, Polypore, stinkhorn, Morels, jelly) [4-5].

The very first crop detection algorithm was proposed in 1996 [6-7]. Infrared images were considered to segment crops and weeds using hysteresis umbral and the Min Neighbouring algorithm for crops planted row wise. New possibilities of solutions started to be adoptable for these similar problems with innovative advancements of ML. CNN based feature detection DBSCAN method applied using Harris corner detection technique in [8] and obtained an accuracy of $98 \%$. Machine learning and deep learning methods are successfully adopted in recognition and classification of plant spices [9]. Indian government is also funding on artificial Intelligence related projects with projected annual GDP growth of $7-7.5 \%$ by 2022 . The improvement's in these technologies has eliminated traditional manual investigation and analysis of feature maps. The deep learning methods will automatically learn and adopt multi-layer processing approach for spatial and temporal data set. This paper is simple means to provide solution for examining mushroom edibility with CNN.

The specific objective of proposed work is to develop an automated image categorization as edible or non-edible mushroom applying deep CNN technique. Currently, CNN is at stage of state-of-art in image 
recognition. $\mathrm{CNN}$ involves classification through learning for this reason large set of images are required to carry on experiment. The obtained images require preprocessing to yield with normalized image set having same size and shape as CNN expect pre specified input sized images. As images are provided as input location and odor cannot be used to classify mushroom. The model is capable of covertly extract features influential to activation function required from the input image through convolution layer. The problem is considered as simple binary class resulting in two feature mining as edible or non-edible.

This research studies on mushroom classification for CNN architectures like sNet, LeNet, AlexNet and cNet. sNet is scalable CNN includes three convolution followed by fully connected layer[10]. LeNet was proposed by Yann Lecun in 1998, it is a simple convolution neural network [11]. LeNet architecture consist of two sets of convolution and pooling layers, flattening convolutional layer, two fully connected layers and softmax classifier at end. AlexNet contains eight layers initial five are convolution and later three are fully connected layer [12]. CNet includes two convolutions and three fully connected layer [13].

This paper organized as follows; in section II a brief review on deep learning, $\mathrm{CNN}$ and its application for mushroom is discussed. The dataset and model description of CNN is included in section III. Proposed work is briefed in section IV followed by experiment discussion and conclusion in section $\mathrm{V}$ and $\mathrm{VI}$ respectively.

\section{RELATED STUDIES}

Deep learning (DL) is descendant of artificial neural network but includes deeper neural nets [14]. DL is an emerging new modern approach finding success in all fields where is being used through its potential applications [15]. The DL model includes more capabilities of real-life system which helps to design better trained model for classification accuracy [16]. The survey reveals that most of works apply convolution neural network with ANN with different levels of convolution layers for image related applications to resolve problem of classification, prediction, or identification $[14,17]$.

Leaf disease detection of thirteen different types of plants is predicted using $\mathrm{CNN}$ in [17]. CNN is widely applied in agricultural field for many purposes. GoogleNet applied for plant disease detection for 14 crops species in [18] and specifically for banana in [19]. For land cover classification is carried on in [14]. Crop type classification among wheat, maize, soybean, sunflower and sugarbeet in [20], similar concept is applied for plant identification considering seven plants in [21] and 44 different plants in [22] for Alex net framework. Few works of CNN evident are corn crop yield estimation in [23], fruit counting of tomatoes [24], apples and oranges [Chen], mangoes, almonds and apples [25]. Cattle race classification is carried on using CNN among five races in [26].

Mushroom is having commercial value because of its nutritional and commercial benefits [1,7]. Mushroom accounts to 0.14 million worldwide among these 7000 are edible but remaining 14000 are not easily recognizable as edible or poisonous $[3,6]$. The machine learning supervised, semi-supervised or unsupervised methods are applied for classification problems in several research works. In [31] classification of mushroom is compared applying multi-layer preceptor (MLP) and base radical network (BRF) among them MLP results are better than BRF. MLP based forecasting tool is presented in [32]. Harvesting of mushroom using Robot vision system applied to check damage through support vector machine (SVM). UCI repository for clustering with $\mathrm{K}$-modes is applied in [30] to analyse different combination of attributes to group mushrooms as poisonous or edible. The Kaggle dataset is used but attributes considered is confusing and less so the accuracy is less. With usage of images in classification accuracies will increase as segmentation will get increased scale and minimize the negative error impacts significantly at large scales [33].

The mushroom Diagnosis Assistance system (MDAS) presented in [31-35], inculcates web application, unified database and mobile phone application. The comparative study is made between Naïve Bayes and decision tree (DT) algorithm for mushroom classification. SVM and Navie bayes algorithms are compared for classification of mushroom in [34] among techniques simulated, SVM ends in better results. Three algorithms decision tree algorithm, SVM, and ANN with clustering are compared in classification of mushroom in [36-38] among them ANN performs better. MLP model is used to create, predict and classify by conducting experiment on dataset of 8124 for training and testing [36], sigmoidal function is used as activation function. K-means, a traditional clustering algorithm is used to categorize dataset of mushroom [39-42]. DT and Navie Bayes are applied on application system named Mushroom Diagnosis Assistance System for classification in [42-46]. The same experiments of [1] are conducted on same dataset in [47-49] using Weka mining tool for interactive identification of 
mushrooms. Multi-nominal problem of classifying problem to multiple categorizes with Navie Bayes, RIDOR and SMO algorithms are proposed in [50-53] amongst Navie Bayes gives best result. Zero, Naïve Bayes and Bayes net methods are applied to classify mushroom in [54,55] as poisonous or non-poisonous and Navie Bayes accuracy is good in simulated results.

\section{MATERIALS AND METHODS}

This section briefs on data preprocess and CNN algorithm which is used in classification in the proposed work.

\subsection{Data retrieval and transformation}

The images used for experimentation is collected

from sources http://www.mushroom.world and
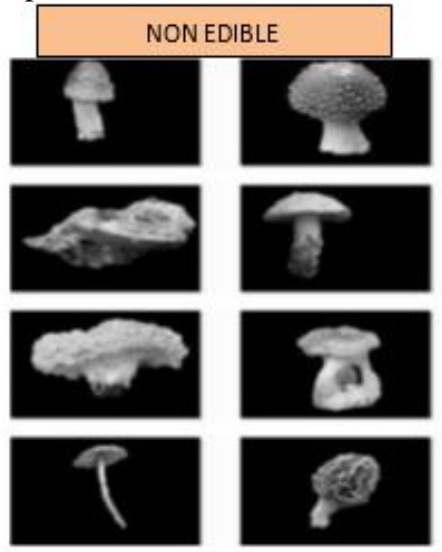
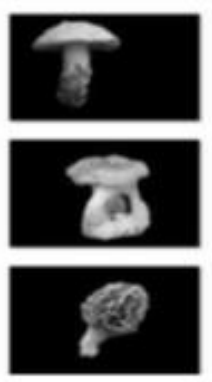
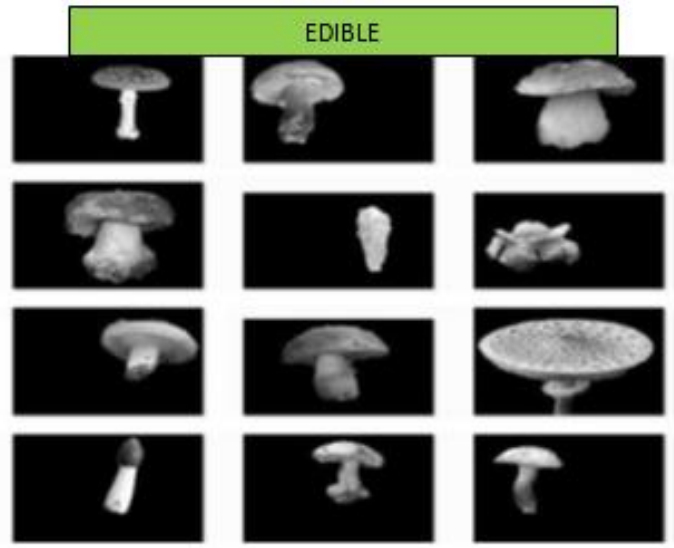

Figure 1 Sample Dataset of mushroom

\subsection{Convolution Neural Network}

The basic layers of the CNN include the convolution, max-pooling and fully-connected (dense) layer [23, 24]. Typically, the network tends to learn better as the network gets deeper [24]. However, this may affect the computational time. Hence, we have carefully designed the network architecture which requires shorter computational time. The highest classification performance is obtained with parameters which are finely tuned during the training phase.

The convolutional layer convolves with the input signal using a kernel (window) [25, 26]. A feature map for the next layer is generated after the convolution. After which, the batch normalization layer is applied to normalize the input training data to flow between the intermediate layers. The purpose is to enable faster learning and boosting. Then, the rectified linear unit is applied to threshold the input data and reduced the redundancies in the data. To reduce the size of the feature map, the max-pooling layer is used. Finally, every neuron of the max-pooling layer is connected to every neuron in the fully-connected layer where the output predicts the outcome (normal or PD) of the input signal [27, 28, 29].

\section{PROPOSED WORK}

CNN architecture is preferably suitable for implementation of model which includes train and test among given dataset. In the proposed work classification of mushrooms into edible or non-edible is performed using CNN architecture and named as DCNN model as in previous study $[13,26]$. Image dataset of mushrooms are classified as edible or non-edible applying machine learning algorithms such as sNet, LeNet, AlexNet, cNet 
and DCNN. Classification is performed using different CNN network architectures and evaluated for performance accuracy. The samples consist of $280 \mathrm{X} 280$ size, preprocessing and normalization for images will be performed. Later, the implementation of DNN model to predict is carried using open source tool Tensorflow. The detailed network architecture of DCNN model is presented in next sub-section.
CNN consist of 4 transformation layers which includes three consecutive convolution and pooling layer pair and at end one fully functional connected dense layer. The complete network structure is pictured in figure. Details of layer input and output array sizes of DCNN shown in figure 2. The network structure and filter size details of sNet, LeNet, AlexNet, cNet and DCNN are included in Table 1 to 5 respectively.

\subsection{Network Structure}

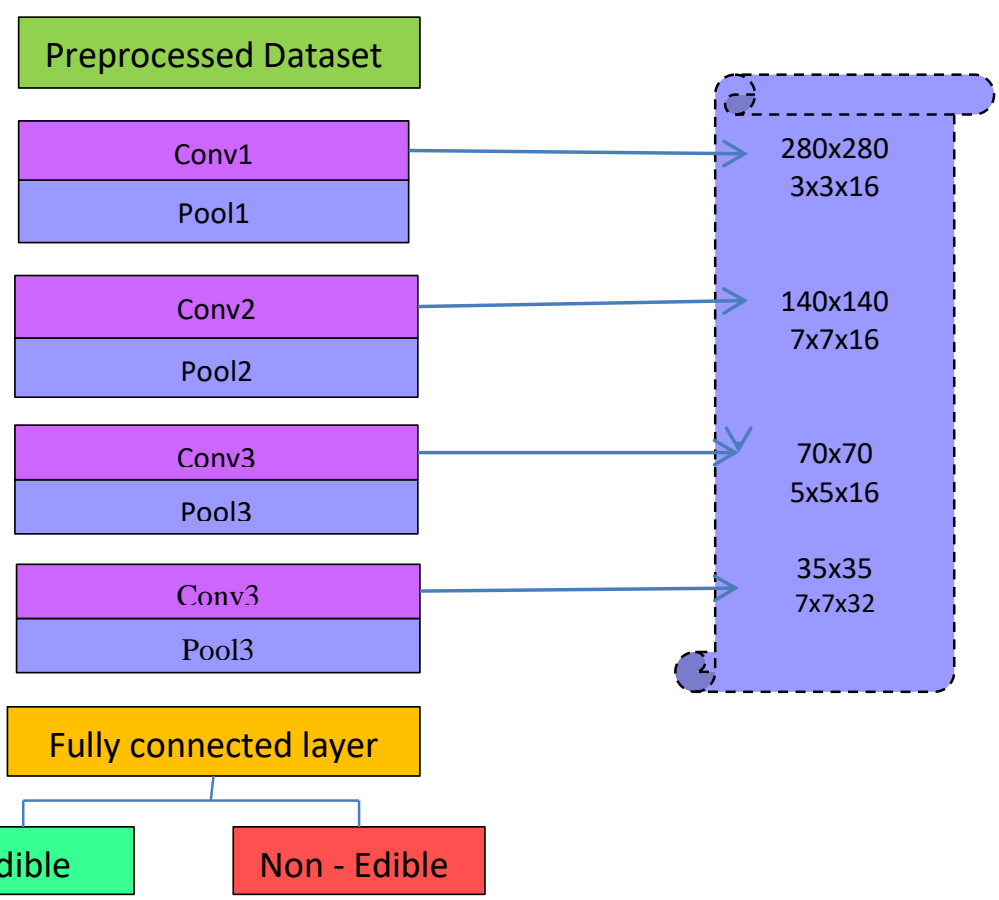

Figure 2 DCNN Network Structure

Table 1. sNet Network structure

\begin{tabular}{|c|l|l|l|l|}
\hline \multicolumn{5}{|c|}{ sNet } \\
\hline Feature & $\begin{array}{l}\text { Convolution } \\
\text { Layer 1 }\end{array}$ & $\begin{array}{l}\text { Convolution } \\
\text { layer 2 }\end{array}$ & $\begin{array}{l}\text { Convolution } \\
\text { layer 3 }\end{array}$ & $\begin{array}{l}\text { Fully } \\
\text { connected } \\
\text { layer }\end{array}$ \\
\hline Input Size & $64 X 64$ & $32 \mathrm{X} 32$ & $16 \mathrm{X} 16$ & $1080 \mathrm{X} 1$ \\
\hline Filter Size & $8 \mathrm{X} 8$ & $4 \mathrm{X} 4$ & $4 \mathrm{X} 4$ & 1024 \\
\hline Activation Function & reLu & reLu & reLu & softmax \\
\hline
\end{tabular}

Table 2. LeNet Network structure

\begin{tabular}{|c|l|l|l|}
\hline \multicolumn{4}{|c|}{ LeNet } \\
\hline Feature & $\begin{array}{l}\text { Convolution } \\
\text { Layer 1 }\end{array}$ & $\begin{array}{l}\text { Convolution } \\
\text { layer 2 }\end{array}$ & $\begin{array}{l}\text { Fully } \\
\text { connected } \\
\text { layer }\end{array}$ \\
\hline Input Size & $32 X 32$ & $10 X 10$ & $10284 X 1$ \\
\hline Filter Size & $5 X 5$ & $2 X 2$ & 1024 \\
\hline Activation Function & Tanh & Tanh & softmax \\
\hline
\end{tabular}


Table 3. cNet Network structure

\begin{tabular}{|c|c|c|c|c|c|}
\hline \multicolumn{6}{|c|}{ cNet } \\
\hline Feature & $\begin{array}{c}\text { Convolution } \\
\text { Layer } 1\end{array}$ & $\begin{array}{c}\text { Convolution } \\
\text { layer } 2\end{array}$ & $\begin{array}{c}\text { Fully } \\
\text { connected } \\
\text { layer }\end{array}$ & $\begin{array}{c}\text { Fully } \\
\text { connected } \\
\text { layer }\end{array}$ & $\begin{array}{c}\text { Fully } \\
\text { connected } \\
\text { layer }\end{array}$ \\
\hline Input Size & $55 \times 55$ & $26 \times 26$ & $4096 X 1$ & $4096 X 1$ & $1000 X 1$ \\
\hline Filter Size & $7 \times 7$ & $3 \times 3$ & 400 & 120 & 84 \\
\hline Activation Function & reLu & reLu & reLu & reLu & softmax \\
\hline
\end{tabular}

Table 4. AlexNet Network structure

\begin{tabular}{|c|c|c|l|l|l|l|l|l|}
\hline \multicolumn{2}{|c|}{ AlexNet } \\
\hline Feature & $\begin{array}{l}\text { Convolut } \\
\text { ion } \\
\text { Layer 1 }\end{array}$ & $\begin{array}{l}\text { Convolution } \\
\text { Layer 2 }\end{array}$ & $\begin{array}{l}\text { Convolution } \\
\text { Layer 3 }\end{array}$ & $\begin{array}{l}\text { Convolution } \\
\text { Layer 4 }\end{array}$ & $\begin{array}{l}\text { Convolution } \\
\text { Laye--r 5 }\end{array}$ & $\begin{array}{l}\text { Fully } \\
\text { connected } \\
\text { layer 1 }\end{array}$ & $\begin{array}{l}\text { Fully } \\
\text { connected } \\
\text { layer 2 }\end{array}$ & $\begin{array}{l}\text { Fully } \\
\text { connected } \\
\text { layer 3 }\end{array}$ \\
\hline Input Size & $64 X 64$ & $32 X 32$ & $16 X 16$ & $8 X 8$ & $4 X 4$ & $4096 X 1$ & $1000 X 1$ & $1000 X 1$ \\
\hline Filter Size & $11 X 11$ & $5 X 5$ & $3 X 3$ & $3 X 3$ & $3 X 3$ & 4096 & 1000 & 84 \\
\hline Activation Fun. & Relu & Relu & Relu & Relu & Relu & Relu & Relu & Soft Max \\
\hline
\end{tabular}

Table 5. DCNN Network structure

\begin{tabular}{|c|c|c|c|c|c|}
\hline \multicolumn{7}{|c|}{ DCNN } & $\begin{array}{c}\text { Convolution } \\
\text { layer 4 }\end{array}$ & $\begin{array}{c}\text { Fully Connected } \\
\text { Layer }\end{array}$ \\
\hline Input Size & $280 X 280$ & $140 X 140$ & $70 X 70$ & $35 X 35$ & $10368 X 1$ \\
\hline Filter Size & $3 X 3$ & $7 X 7$ & $5 X 5$ & $7 X 7$ & 1024 \\
\hline Activation Function & Relu & Relu & Relu & Relu & Softmax \\
\hline
\end{tabular}

\subsection{Design and Implementation}

The model input is matrix form images values preprocessed as input range between 0 and 1. Input is assumed to be inputted as in eq 1 . In the eq. $1 \mathrm{~s}$ is the sample index. Total number of samples is $\mathrm{S}$ in size (15400). $\mathrm{N}$ and $\mathrm{M}$ is total number of sweeps in one sample (280) and resolution channels captured bands (280) respectively. Features are extracted through CNN with combination of convolution and pooling layers. In model pooling convolution combination layer works as in eq. 2 for convolution layer 1 . In eq. $2 \mathrm{~W}$ is activation function, $\mathrm{c}_{1}$ is number of convolution filters, $\mathrm{b}$ is weight and bias. Activation function isincluded with the aim to manage, transform, scale and output data range to train model. ReLu function is operated as in eq 3 for the activation function. It includes complex and non-linear functions. Similarly, if framework consists of $n$th convolution layer then output will be generated using eq 4. In the eq .4, total number of proposed CNN architecture is indicated with T, Extraction of feature in $\mathrm{CNN}$ is performed at two stages on using combination of convolution and pooling. Next feature extraction using dense fully functional layer. The dense fully functional layer is computed as in eq 5 . In the eq 5 indicates leveling function to learn features extracted in convolution layer. The extraction of required attributes of proposed model is obtained using total fully functional layer of eq 6.In the eq 6 , weight and bias parameters are taken into considered in computation $W_{N}$ and $b_{N}$ respectively. The $E_{f s}$ fully functional layer generates on predicted output from the $\mathrm{S}$ samples.

$$
\begin{aligned}
& X_{s}=\left[x_{i, j}, \ldots \ldots \ldots \ldots \ldots . x_{N, M}\right] \quad i \in[1, N], j \in[1, M], s \in[1, S] \\
& c p_{1}=\operatorname{pool}\left(\sigma\left(W_{1}^{f} * X_{s}+b_{1}^{f}\right)\right) \quad f \in\left[1, c_{1}\right] \\
& \sigma(x)= \begin{cases}x & \text { if } x>0 \\
0 & \text { Otherwise }\end{cases} \\
& o_{n}=\operatorname{pool}\left(\sigma\left(W_{n}^{f} * O_{n-1}+b_{n}^{f}\right)\right), f \in\left[1, c_{n}\right], n \in[1, T-1]
\end{aligned}
$$




$$
\begin{aligned}
& O^{l}=f\left(O_{n-1}\right) \\
& E_{f s}=W_{N} *\left(f\left(\operatorname{pool}\left(\sigma\left(W_{n}^{f} * O_{n-1}+b_{n}^{f}\right)\right)\right)\right)+b_{N}
\end{aligned}
$$

\section{RESULT AND DISCUSSION}

The classification of mushroom dataset is performed using $\mathrm{CNN}$ as mentioned. Proposed CNN model is developed using Keras in python. The classification is evaluated using accuracy, sensitivity and specificity evaluation parameters. Performance of classification is analysed through comparison in two stages. In first stage deep CNN, SVM, Navie Bayes, random forest, decision tree and KNN tested for classification of mushrooms. In second stage test, deep CNN, LeNet, cNet, sNet and AlexNet are compared. The result obtained is represented in figure 3 .

The CNN is also known for its multiple network architectures. The different CNN framework DCNN, LeNet, cNet, sNet and AlexNet used in performance analysis. Classification process of these frameworks is tabulated in table 6. among the network architecture DCNN perform better than others.

The machine learning algorithms were compared for classification with dataset split of $70 \%$ test and $30 \%$ train and results are plotted as in figure 4 . The comparison result is tabulated in table 7 . In order to find better performance in each method best parameters are set accordingly. Deep CNN method result is better in terms of accuracy, sensitivity and specificity. Results of SVM, Naive Bayes, random forest and $\mathrm{KNN}$ are getting results next to DCNN. SVM and Naive Bayes are better than random forest, decision tree and $\mathrm{KNN}$ in results and training time required might be because of large dataset. Among all methods DNN and SVM took more training and computational time.

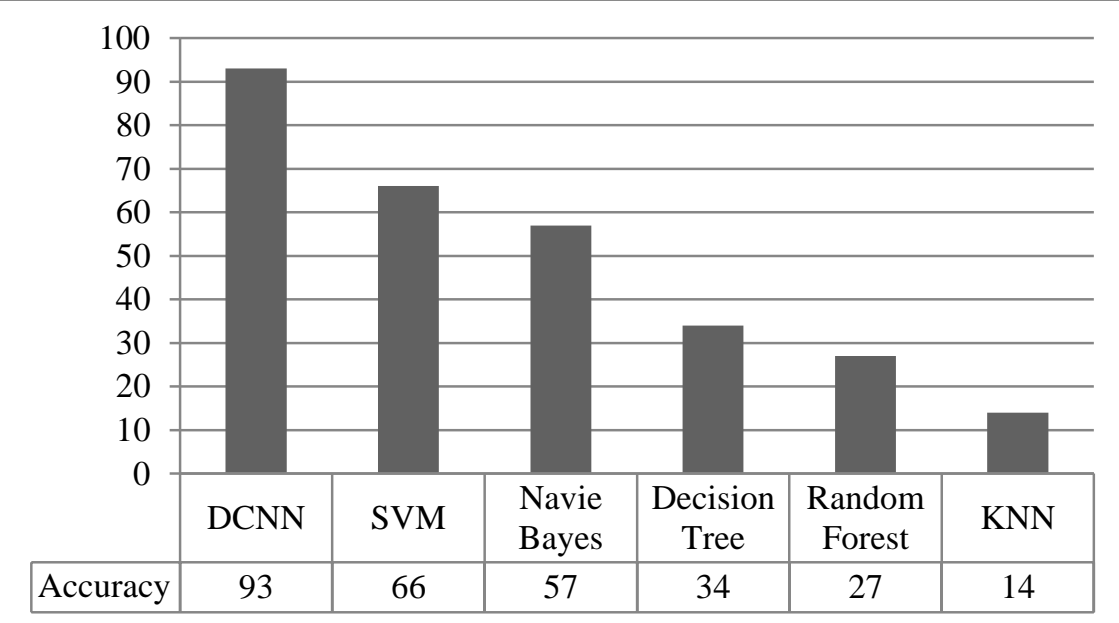

Figure 3 Comparison results for edibility

Table 6. Network models Framework of Classification

\begin{tabular}{|c|c|c|c|c|c|}
\hline Parameters & Input size & $\begin{array}{c}\text { Layer } \\
\text { numbers }\end{array}$ & $\begin{array}{c}\text { Number of } \\
\text { parameters }\end{array}$ & Iterations & Accuracy \\
\hline sNet & $64 \times 64$ & 4 & 135872 & 100 & 80.4 \\
\hline LeNet & $32 \times 32$ & 9 & 652500 & 100 & 86.48 \\
\hline AlexNet & $64 \times 64$ & 11 & 20166688 & 100 & 93.86 \\
\hline cNet & $64 \times 64$ & 8 & 6421568 & 100 & 96.86 \\
\hline DCNN & $280 \times 280$ & 16 & 1659376 & 100 & 97.03 \\
\hline
\end{tabular}



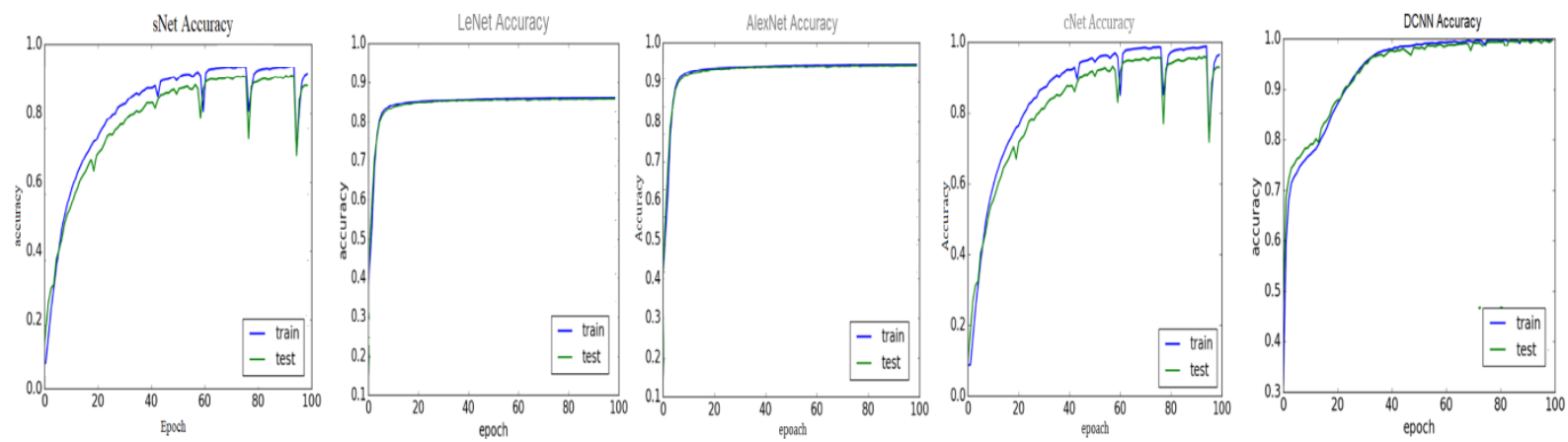

Figure 4 Classification results obtained in different models at each epoch.

Table 7: Comparison of classification results

\begin{tabular}{|c|c|c|c|c|c|c|}
\hline Method & DCNN & SVM & $\begin{array}{c}\text { Naïve } \\
\text { Bayes } \\
\text { Metric }\end{array}$ & $\begin{array}{c}\text { Random } \\
\text { forest }\end{array}$ & $\begin{array}{c}\text { Decision } \\
\text { tree }\end{array}$ & KNN \\
\hline Accuracy & 0.93 & 0.66 & 0.57 & 0.34 & 0.27 & 0.14 \\
\hline Sensitivity & 0.91 & 0.57 & 0.57 & 0.37 & 0.28 & 0.14 \\
\hline Specificity & 0.90 & 0.66 & 0.6 & 0.4 & 0.27 & 0.14 \\
\hline
\end{tabular}

\section{CONCLUSION}

In proposed work $\mathrm{CNN}$ model classifies mushroom either edible or not. The proposed method of DCNN proves to be better in classifying mushroom with an accuracy of 0.93 . Along with this conclusion can be drawn that the depth of network and performance of classification not correlated so in that case with increase in complexity will not yield any better results. Further investigate on accuracy improvements can be tested through improved deep learning models. The optimal depth and size of filters can be researched.

\section{REFERENCES}

[1] Rial Adity and Setia Hadi Purwono, Jamur - Info Lengkap dan Kiat Sukses Agribisnis. Depok, Indonesia/West Java: Agriflo, 2012.

[2] Kristianus Sunarjon Dasa, "Pemanfaatan bagas sebagai campuran media pertumbuhan jamur tiram putih," vol. 11, pp. 195-201, 2011.

[3] N. Zahan, M. Z. Hasan, M. A. Malek and S. S. Reya, "A Deep Learning-Based Approach for Edible, Inedible and Poisonous Mushroom Classification," 2021 International Conference on Information and Communication Technology for Sustainable Development (ICICT4SD), 2021, pp.
440-444,

doi: 10.1109/ICICT4SD50815.2021.9396845.

[4] Al-Mejibli and D. Hamed Abd, "Mushroom Diagnosis Assistance System Based on Machine Learning by Using Mobile Devices Intisar Shadeed Al-Mejibli University of Information Technology and Communications Dhafar Hamed Abd Al-Maaref University College," vol. 9, no. 2, pp. 103-113, 2017.https://doi.org/10.29304/jqcm.2017.9.2.319

[5] M. Alameady, "Classifying Poisonous and Edible Mushrooms in the Agaricus," International Journal of Engineering Sciences \& Research Technology, vol. 6, no. 1, pp. 154-164, 2017.

[6] Duong, L.T.; Nguyen, P.; Di Sipio, C.; Di Ruscio, D. Automated fruit recognition using EfficientNet and MixNet. Comput. Electron. Agric. 2020, 171, 105326.

[7] J. M. J. Ward, E. L. Stromberg, D. C. Nowell, F. W. Jr. Nutter, "Gray leaf spot: a disease of global importance in maize production," Plant Disease, vol. 83, no. 10 , pp. 884-895, Oct. 1999.

[8] G. Wang, Y. Sun, J. X. Wang, "Automatic ImageBased Plant Disease Severity Estimation Using Deep Learning," Computational Intelligence and Neuroscience, vol. 2017, no. 2, pp. 1-8, Jul. 2017 
[9] Schmidhuber J (2015) Deep learning in neural networks: an overview. Neural Networks 61, 85-117.

[10] Izza SabillaS, Sarno R, Siswantoto J, Estimating concentration using Artificial neural network for electronic .Proceds compu. Sci. 2017, 124, 181-188

[11] Sven Behnke, N B Karayiannis, CNet competitive neuraltrees for pattern classification, IEEE transaction son neural network, 9, 6, 1996

[12] Phongsakhon Tongcham, Pichaya Supa, Peerapong Pornwongthong, Pitcha Prasitmeeboon, Mushroom spawn quality classification with machine learning, Computers and Electronics in Agriculture, Vol. 179, 2020, 105865, ISSN 0168-1699, https://doi.org/10.1016/j.compag.2020.105865.

[13] Peng P, Xiaofang P, Classification using deep convolution neural networks, Sensors, 2018,18, 157.

[14] Wang, P.; Liu, J.; Xu, L.; Huang, P.; Luo, X.; Hu, Y.; Kang, Z. Classification of Amanita Species Based on Bilinear Networks with Attention Mechanism. Agriculture 2021, 11, 393. https://doi.org/ 10.3390/agriculture 11050393

[15] Schmidhuber J, Deep learning in neural networks: an overview. Neural Networks 61, 85-117, 2015.

[16] Najafabadi MM, Villanustre F, Khoshgoftaar TM, Seliya N, Wald R and Muharemagic E (2015) Deep learning applications and challenges in big data analytics. Journal of Big Data 2, 1

[17] Sladojevic S, Arsenovic M, Anderla A, Culibrk D and Stefanovic D (2016 Deep neural networks based recognition of plant diseases by leaf image classification. Computational Intelligence and Neuroscience 2016, 3289801.

[18] Mohanty SP, Hughes DP and Salathé M (2016) Using deep learning for image-based plant disease detection. Frontiers in Plant Science 7, 1419.doi: 10.3389/fpls.2016.01419.

[19] Amara J, Bouaziz B and Algergawy A (2017) A deep learning-based approach for banana leaf diseases classification. In Mitschang B (ed.),Datenbanksysteme für Business, Technologie und Web (BTW 2017) -Workshopband. Lecture Notes in Informatics (LNI). Stuttgart, Germany:Gesellschaft für Informatik, pp. 79-88.

[20] Kussul N, Lavreniuk M, Skakun S and Shelestov A (2017) Deep learning classification of land cover and crop types using remote sensing data.IEEE Geoscience and Remote Sensing Letters 14, 778-782.
[21] Grinblat GL, Uzal LC, Larese MG and Granitto PM (2016) Deep learning for plant identification using vein morphological patterns. Computers and Electronics in Agriculture 127, 418-424.

[22] Kuwata K and Shibasaki R (2015) Estimating crop yields with deep learning and remotely sensed data. In IEEE International Geoscience and Remote Sensing Symposium (IGARSS). Milan, Italy: IEEE, pp. 858-861.

[23] Rahnemoonfar M and Sheppard C (2017) Deep count: fruit counting based on deep simulated learning. Sensors 17, 905.

[24] Chen SW, Shivakumar SS, Dcunha S, Das J, Okon E, Qu C, Taylor CJ andKumar V (2017) Counting apples and oranges with deep learning: a datadriven approach. IEEE Robotics and Automation Letters 2, 781-788

[25] Bargoti S and Underwood J (2017) Deep fruit detection in orchards. In Okamura A (ed.), 2017 IEEE International Conference on Robotics and Automation (ICRA). Piscataway, NJ, USA: IEEE, pp. 3626-3633.

[26] Santoni MM, Sensuse DI, Arymurthy AM and Fanany MI (2015) Cattle race classification using gray level co-occurrence matrix convolutional neural networks. Procedia Computer Science 59, 493-502.

[27] . P. Ratchapra, and C. Chantrapornchai, "Expert system for rice disease diagnosis," E-Journal SU, vol. 6, no. 1, 2013.

[28] Kononenko, "Machine learning for medical diagnosis: history, state of the art and perspective," Artificial Intelligence in medicine, vol. 23, no. 1, pp. 89-109, 2001.

[29] Roni Hamonangan, Meidika Bagus Saputro, Cecep Bagus Surya Dinata Karta Atmaja, "Accuracy of classification poisonous or edible of mushroom using naïve bayes and K-nearest neighbors", J. Soft. Comp. Explor., Vol. 2, No. 1, March 202, pp. 53 - 60

[30] Onuodu F E, K-Modes Clustering Algorithm in Solving Data Mining Problems for Mushroom Dataset, Semantic Scholar, 2015.

[31] Cáceres M.N., González Arrieta M.A. (2013) Automatic Prediction of Poisonous Mushrooms by Connectionist Systems. In: Omatu S., Neves J., Rodriguez J., Paz Santana J., Gonzalez S. (eds) Distributed Computing and Artificial Intelligence. Advances in Intelligent Systems and Computing, vol 217.Springer, Cham. 
[32] Tim Kovacs, Strength or Accuracy : Credit Assignment in Learning Classifier Systems, Springer, 2004, pp.2-3.

[33] Zengyou He, XiaofeiXu, Shengchun Deng, A Cluster Ensemble Method for Clustering Categorical Data, Information Fusion, 6(2), June 2005, pp.143-151.

[34] AlirezaMasoudian, Kenneth A. Mcisaac , Application of Support Vector Machine to Detect Microbial Spoilage of Mushrooms, International Conference on Computer and Robot Vision (CRV), 28-31 May 2013, 30 July 2013.

[35] P. Kumar, V. K. Sehgal, D. S. Chauhan, and others, "A benchmark to select data mining based classification algorithms for business intelligence and decision support systems," arXiv preprint arXiv:1210.3139,

2012.

https://doi.org/10.5121/ijdkp.2012.2503

[36] M. Alameady, "Classifying Poisonous and Edible Mushrooms in the Agaricus," International Journal of Engineering Sciences \& Research Technology, vol. 6,no. 1, pp. 154-164, 2017.

[37] F. E. Onuodu, "K-Modes Clustering Algorithm in Solving Data Mining Problems for Mushroom Dataset," nternational Journal of Advanced Research in Computer Science and Software Engineering, vol. 5, no. 9, pp. 596-603, 2015.

[38] Al-Mejibli and D. Hamed Abd, "Mushroom Diagnosis Assistance System Based on Machine Learning by Using Mobile Devices Intisar Shadeed Al- Mejibli University of Information Technology and Communications Dhafar Hamed Abd Al-Maaref University College," vol. 9, no. 2, pp. 103-113, 2017.https://doi.org/10.29304/jqcm.2017.9.2.319

[39] Chang-yanab, Z. Ji-xian, and L. Zheng-jun, "Study on methods of noise reduction in a stripped image," The International Archives of the Photogrammetry, Remote Sensing and Spatial Information Sciences, vol. XXXVII. Pa, no. 1, pp. 2-5, 2008

[40] Chowdhury and S. Ojha, "An Empirical Study on Mushroom Disease Diagnosis : A Data Mining Approach," International Research Journal of Engineering and Technology(IRJET), vol. 4, no. 1, pp.529-534, 2017.

[41] S. Beniwal and B. Das, "Mushroom Classification Using Data Mining Techniques," International Journal of Pharma and Bio Sciences, vol. 6, no. 1, pp. 1170-1176, 2015

[42] K. Yu, L. Tan, L. Lin, X. Cheng, Z. Yi and T. Sato, "Deep-Learning-Empowered Breast Cancer Auxiliary
Diagnosis for 5GB Remote E-Health," IEEE Wireless Communications, vol. 28, no. 3, pp. 54-61, June 2021, doi: 10.1109/MWC.001.2000374.

[43] K. Yu, Z. Guo, Y. Shen, W. Wang, J. C. Lin, T. Sato, "Secure Artificial Intelligence of Things for Implicit Group Recommendations", IEEE Internet of Things Journal, 2021, doi: 10.1109/JIOT.2021.3079574.

[44] L. Zhen, A. K. Bashir, K. Yu, Y. D. Al-Otaibi, C. H. Foh, and P. Xiao, "Energy-Efficient Random Access for LEO Satellite-Assisted 6G Internet of Remote Things", IEEE Internet of Things Journal, doi: 10.1109/JIOT.2020.3030856.

[45] L. Tan, K. Yu, F. Ming, X. Cheng, G. Srivastava, "Secure and Resilient Artificial Intelligence of Things: a HoneyNet Approach for Threat Detection and Situational Awareness", IEEE Consumer Electronics Magazine, 2021, doi: 10.1109/MCE.2021.3081874.

[46] Z. Guo, A. K. Bashir, K. Yu, J. C. Lin, Y. Shen, "Graph Embedding-based Intelligent Industrial Decision for Complex Sewage Treatment Processes", International Journal of Intelligent Systems, 2021, doi: 10.1002/int.22540.

[47] Z. Guo, K. Yu, A. Jolfaei, A. K. Bashir, A. O. Almagrabi, and N. Kumar, "A Fuzzy Detection System for Rumors through Explainable Adaptive Learning", IEEE Transactions on Fuzzy Systems, doi: 10.1109/TFUZZ.2021.3052109.

[48] Puttamadappa, C., and B. D. Parameshachari. "Demand side management of small scale loads in a smart grid using glow-worm swarm optimization technique." Microprocessors and Microsystems 71 (2019): 102886.

[49] Rajendrakumar, Shiny, and V. K. Parvati. "Automation of irrigation system through embedded computing technology." In Proceedings of the 3rd International Conference on Cryptography, Security and Privacy, pp. 289-293. 2019.

[50] Kumar, M. Keerthi, B. D. Parameshachari, S. Prabu, and Silvia liberata Ullo. "Comparative Analysis to Identify Efficient Technique for Interfacing BCI System." In IOP Conference Series: Materials Science and Engineering, vol. 925, no. 1, p. 012062. IOP Publishing, 2020.

[51] Nguyen, Tu N., Bing-Hong Liu, Nam P. Nguyen, and Jung-Te Chou. "Cyber security of smart grid: attacks and defenses." In ICC 2020-2020 IEEE International Conference on Communications (ICC), pp. 1-6. IEEE, 2020. 
[52] Hu, Liwen, Ngoc-Tu Nguyen, Wenjin Tao, Ming C. Leu, Xiaoqing Frank Liu, Md Rakib Shahriar, and SM Nahian Al Sunny. "Modeling of cloud-based digital twins for smart manufacturing with MT connect." Procedia manufacturing 26 (2018): 11931203.

[53] Le, Ngoc Tuyen, Jing-Wein Wang, Duc Huy Le, Chih-Chiang Wang, and Tu N. Nguyen. "Fingerprint enhancement based on tensor of wavelet subbands for classification." IEEE Access 8 (2020): 6602-6615.

[54] Bhuvaneswary, N., S. Prabu, K. Tamilselvan, and K. G. Parthiban. "Efficient Implementation of Multiply Accumulate Operation Unit Using an Interlaced Partition Multiplier." Journal of Computational and Theoretical Nanoscience 18, no. 4 (2021): 13211326.

[55] Prabu, S., Balamurugan Velan, F. V. Jayasudha, P. Visu, and K. Janarthanan. "Mobile technologies for contact tracing and prevention of COVID-19 positive cases: a cross-sectional study." International Journal of Pervasive Computing and Communications (2020). 